\title{
Assessment of Battery Ageing and Implementation of an Ageing Aware Control Strategy for a Load Leveling Application of a Lithium Titanate Battery Energy Storage System
}

\author{
Emil Namor, Dimitri Torregrossa, Fabrizio Sossan, Rachid Cherkaoui, Mario Paolone \\ Ecole Polytechnique Fédérale de Lausanne \\ Lausanne, Switzerland \\ Email: emil.namor@epfl.ch
}

\begin{abstract}
The manuscript describes a method to embed into a battery energy storage system (BESS) control strategy the performance degradation associated with the battery operation. In particular, the proposed method aims at minimizing the degradation of the BESS electrochemical cells. A load leveling strategy is described as a case study and the ageing effects associated with the battery current extraction are embedded as constraints into the optimization problem. The main contributions of the work, compared to the existing literature are: i) the degradation process is formulated as a weighted energy throughput, thus taking into account the C-rate effect on the degradation phenomena; ii) the performance of the proposed control strategy has been applied to a large scale lithium-titanate BESS of $280 \mathrm{kWh}$ interfaced to a $20 \mathrm{kV}$ active distribution network.
\end{abstract}

\section{INTRODUCTION}

In the last decades renewable energy resources (RERs) have gained more and more importance in power systems and this trend is expected to continue in the upcoming years. While being beneficial from an environmental point of view, the increased penetration of RERs in power systems has brought several challenges related to the volatility of these sources. One of the technologies capable of addressing these issues is BESSs. Therefore, in the near future, BESSs are expected to have a diffusion similar to the one of RERs [1] [2] . They are currently used for applications such as peak-shaving [3], ancillary services [4], operation of isolated microgrids [5] or dispatch of portions of the grid [6].

One of the main challenges for a massive and faster penetration of BESSs in power grids is their relatively high cost, when referring to the lifetime of these devices. Graphite based lithium ion batteries, can generally perform up to 30004000 cycles [7] and have a calendar life of about 10-15 years. Battery life management is thus important to make BESS a viable technology for power systems. This issue is being addressed from a chemical perspective, by looking for more life-robust technologies (e.g. lithium-titanate batteries [8]) but need to be tackled as well from a system and control perspective in order to maximize the battery lifetime and optimize BESS usage so that they can deliver relevant services while enduring a minimal degradation. In order to achieve the above goals it is necessary to assess the degradation trends of batteries during their operation and to integrate ageing limitations in the BESS control algorithms [9][10].

978-1-5090-1815-4/16/\$31.00 (C)2016 IEEE
The present work proposes an original way to integrate ageing effetc in the control strategy.

It is well known that the main ageing factors in lithium ion electrochemical cells are: i) the rate at which the cells are charged and discharged (the higher the rate the faster the degradation); ii) the state of charge at which they are kept; iii) the temperature (temperatures considerably higher or lower than the nominal one usually increase ageing) [11]. The current work focuses on the first two ageing stress factors. The effect of the temperature is here ignored as, in most stationary applications, as well as in the current case study, temperature is regulated by an air conditioning systems and kept within suitable bounds around the rated temperature of the cells.

The proposed method take into account the current rate effect through a weighted energy throughput. This method is an extension of the weighted charge throughput proposed in [12]. The paper is structured as follows: section II introduces the proposed ageing assessment method; section III present the experimental setup on which the method has been used, the main results of the assessment of degradation during BESS operation and the implementation of an ageing aware strategy; finally section IV summarizes the main results and the main contribution of this manuscript.

\section{METHODOLOGY}

In this section the procedure for the ageing assessment during BESS operation is described and a method to integrate the results of this procedure in the battery control is described.

\section{A. Weighted Energy Throughput}

The method here described relies on the principle that an eletrochemical cell can exchange a finite amount of charge during its lifespan, as described in [12]. This value can be assessed through cycling tests and calculated as the charge that pass through a cell during a complete discharge-charge cycle (i.e. $2 C_{\text {rated }}$ ) multiplied by the total number of cycles that a battery can perform before depletion. This method, known as charge throughput, has the advantage of relying only on values that are most often given by the manufacturers (rated capacity and number of cycles). It has to be noted that the depth of discharge (DOD) of the cycles is implicitly taken into account in a linear fashion, as a cycle with lower DOD has also proportionally lower charge throughput. Assessment 
methods relying on physical models of the cells and of their degradation phenomena, such as the one proposed in [13] may provide more precise results, but requires the development and validation of complex models and the knowledge of a set of physical parameters usually not available to the end-user. The charge throughput, on the contrary, while being rather simple, neglects a series of factors, such as the influence of the C-rate on the cycling ageing or the calendar ageing. In the following, a method is presented to assess a weighted throughput that takes into account these factors, by using exclusively on data provided by the manufacturer and available on the cell datasheets.

The energy exchanged by a cell during its operation $E_{\text {exch }}$ can be defined as in:

$$
E_{\text {exch }}=\Delta T \sum_{k=1}^{N}|w(k) B(k)|
$$

The ratio between $E_{\text {exch }}$ and the energy exchanged during a complete cycle by the cell, i.e. $2 E_{\text {batt }}$, defines the number of equivalent cycles that the cell has performed in the period $n \Delta T$ :

$$
N_{c}=\frac{E_{\text {exch }(k)}}{2 E_{\text {batt }(k)}}
$$

The denominator of Eq. (2) evolves with time, being the maximum energy that can be stored in the cell defined as:

$$
E_{\text {batt }(k)}=E_{\text {rated }}\left(1-\frac{\sum_{j=0}^{k-1} N_{c}(j)}{N_{M}}\right)
$$

Where $E_{\text {rated }}$ is the rated energy capacity of the cell and $N_{M}$ is the nominal number of cycles that the cell can endure, also usually indicated on the cell data-sheet. In the case of the cells under analysis, it is $N_{M}=20000$ cycles, this value being defined as the number of cycles at $4 \mathrm{C}$ after which the cell capacity falls below $80 \%$ of its initial value. Comparing $N_{c}$ with the maximum number of cycles for the cell $N_{M}$, a time in years until the cell end of life (EOL) can be defined as:

$$
E O L=\frac{N_{M}}{N_{c}} \frac{1}{365}
$$

\section{B. Definition of the weights $w(k)$}

The cells used for the here proposed analysis are $30 \mathrm{Ah}$ lithium-titanate batteries, whose expected cycling life-time is of 20000 cycles. The weights are calculated from cycling data provided by the cell manufacturer on their cell data-sheet. Fig. 1 shows the evolution of the state of health (SOH) of the cell, defined as the cell capacity after a certain time of operation normalized with its initial one, with the number of cycles and with different current rates. Notably, the blue dots and line refer to a cycling performed at $4 \mathrm{C}$, whereas the red ones refer to a $1.5 \mathrm{C}$ cycling.

The data points in Fig. 1 are the capacity measured values for different number of cycles. The lines, are obtained through linear interpolation of the measured data. Their slopes can be used to define the weights for the energy throughput

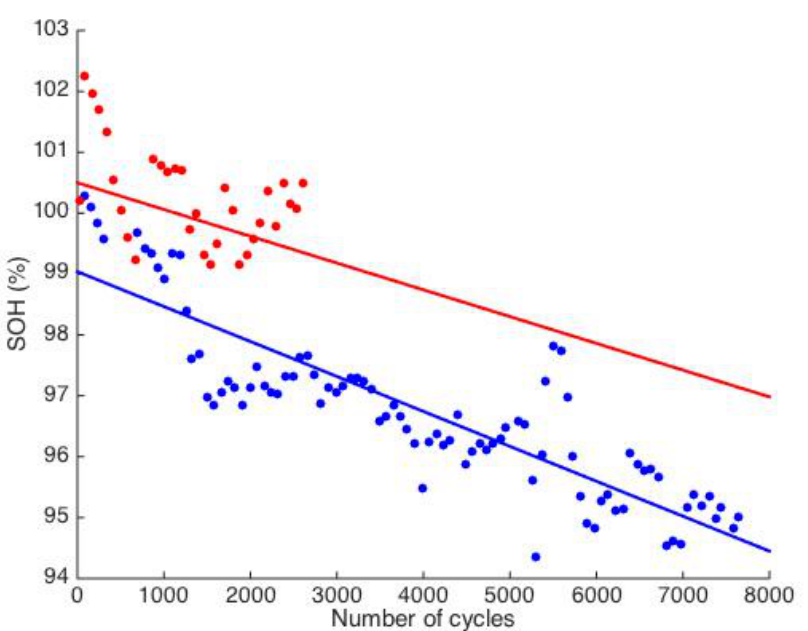

Figure 1. Experimental data from cycling ageing test at $4 \mathrm{C}$ (blue) and $1.5 \mathrm{C}$ (red).

Table I

WEIGHTING FACTORS FOR THE C-RATES IN FIG. 1

\begin{tabular}{llll}
\hline C-rate & $\mathrm{SOH}_{0}$ & $m\left(10^{-4}\right)$ & $w$ \\
\hline $4 \mathrm{C}$ & 100.8 & 5.617 & 1 \\
$1.5 \mathrm{C}$ & 99.11 & 4.117 & 0.73 \\
\hline
\end{tabular}

calculation, and are shown in Table I. In fact, it can be seen that for a higher rate, a higher slope is present (in agreement with the fact that cycling at higher rate causes faster degradation of the cell).

The interpolated lines are defined as:

$$
S O H(N o C)=S O H_{0}-m * N o C
$$

where $N o C$ is the number of cycles, $m=f\left(C_{\text {rate }}\right)$ is the rate of decrease of the state of health, and is function of the $C_{\text {rate }}$ at which the cycles are performed. In Fig. 1, notably, the behaviors for the experimental data and the cycling trends of $1.5 \mathrm{C}$ and $4 \mathrm{C}$ are represented. The weights $w$ are finally obtained by normalizing the values of $m$ on the value at $4 \mathrm{C}$, given that the maximum number of cycles for the examined cell is defined at this particular rate.

The above data allow to extract a trend line for the weights at current rates different from the ones at which the experiments have been performed by the manufacturer. Through linear interpolation it is obtained that:

$$
w\left(C_{\text {rate }}\right)=0.57+0.11 * C_{\text {rate }}
$$

In equation 6 , the constant term is relative to a degradation process independent from the cycling rate whereas the effect of C-rate is found in the second term. This method allows to obtain a weighting factor for any cycling condition encountered in the operation of the BESS. 


\section{TARGETED CASE}

The method described above is used in this section on an setup described in section III-A. Section III-B applies the proposed method to assess how a load leveling strategy [14] [15] [16] implemented with this BESS affect the degradation of the cells. Section III-C, finally, shows how an additional constraint can be added to this strategy to limit ageing and set a lifetime duration objective for the BESS.

The load leveling used as a case study is operating in $24 \mathrm{~h}$ periods and formulated as follows. The feeder forecasted load profile is defined the day ahead of operation through datadriven forecasting. The BESS power injection is then defined so that the overall feeder power profile is leveled. The MV feeder power profile is then dispatched through an intra-day operation of the BESS.

The control strategy for the BESS aims at reducing the deviation of the load profile of a MV feeder $\hat{L}$ from its mean power value $\hat{L}_{a v g}$, through the utilization of the BESS power $B$. For the BESS it is therefore determined the profile of the power injections along a $24 \mathrm{~h}$ period, with a resolution of 5 minutes. The load profile is built through the averaging of forecast scenarios composed by historical data and the problem is solved the day ahead of the operation.

$$
\underset{B_{i}}{\arg \min } \sum_{i=1}^{N=288}\left(\left(\hat{L}_{i}-\hat{L}_{a v g}\right)-B_{i}\right)^{2}
$$

Subject to:

$$
\begin{aligned}
& \left|B_{i}\right|<B_{\max } \\
& \left|P_{i}\right|<P_{\max } \\
& 0<S O E_{i}<1
\end{aligned}
$$

The constraints for this problem, without taking into account for ageing limitations, bound the battery power $B_{i}$, the power absorbed by the feeder $P_{i}=B_{i}+L_{i}$ and the battery state of energy $S_{i} E_{i}$ [17] built as:

$$
S O E_{i}=S O E_{0}+\frac{\Delta T}{C_{n o m}} \sum_{j=0}^{i} B_{j}
$$

$C_{n o m}$ is the nominal capacity of the BESS.

The energy throughput is here calculated on the BESS usage defined in the day-ahead problem and the constraint imposed on the BESS usage described in section III-C limits the loadleveling objective of the control strategy.

\section{A. Case Study Setup}

The methodology described in the previous section has been deployed with a lithium-titanate BESS of $280 \mathrm{kWh}$, connected to a $20 \mathrm{kV}$ active distribution network characterized by a peak load of about $300 \mathrm{~kW}$ and embedding $100 \mathrm{kWp}$ of PV generation [18]. The BESS performs the leveling of the feeder load. The battery power, current and state of charge are measured in real time by a tailored battery management system (BMS) with a sampling rate of one second.

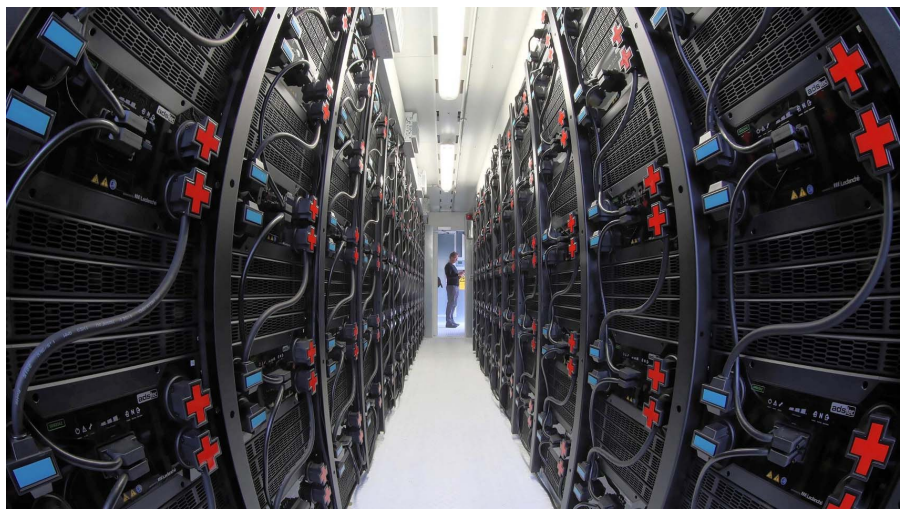

Figure 2. Interior of the lithium-titanate BESS (picture of Alain Herzog, EPFL).

\section{B. Ageing Assessment of the Unconstrained Load Leveling Strategy}

In this section the weighted energy throughput method described and characterized above is used to assess the ageing resulting from the load leveling strategy. Several scenarios obtained from historical data of the power consumption of the targeted feeder are considered. Fig. 3 shows the power and stored energy profiles of the BESS during 24h from one of the scenarios taken into account. In the upper image we can see the behavior of the power of the MV feeder without and with the contribution of the BESS. In the middle figure we can see the daily profile of the BESS power and in the lower figure we can see the daily evolution its stored energy. The power profiles are discretized in 5 minute intervals. The exchanged energy of the BESS can be therefore calculated as the sum of battery power absolute values within $24 \mathrm{~h}$, weighted though the $w$ factors defined in section II-B. This energy can then be compared with the rated energy of a complete cycle to obtain an equivalent number of cycles per day and from this value an estimation of the battery duration before depletion, if in use with this specific control strategy. Table II collects the ageing data obtained for several days of load leveling operation, taken in various moment of the year and of the week.

It can be seen how, for this specific battery chemistry and this specific application, the $E O L$ values are very high, i.e. the ageing of the battery due to its cycling takes place in a very long period. These values are especially high when compared to the calendar lifetime of these devices, which, for this specific BESS is of 20 years. The BESS itself, in such a long time span can incur in ageing from many and various causes, such as calendar and mechanical ageing, defects of individual parts of the BESS or disruptive events. The above analysis, nevertheless, remains extremely important, as it assess the ageing that is directly linked to the battery operation and it assess therefore, if and how the control strategies developed for the BESS need to be aware of this process.

In Table III are reported average daily values for several relevant metrics, obtained from a simulation of 5 years of operation of the BESS. Notably, $\operatorname{var}(L)$ and $\operatorname{var}(P)$ are the 


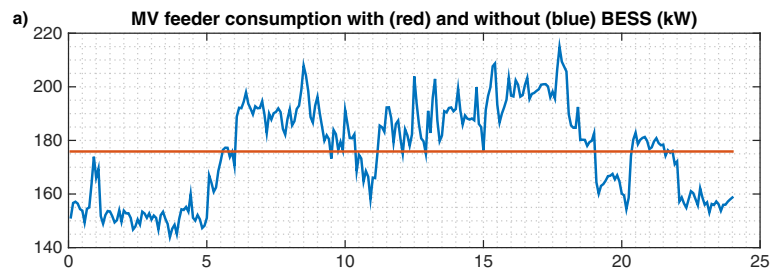

b)
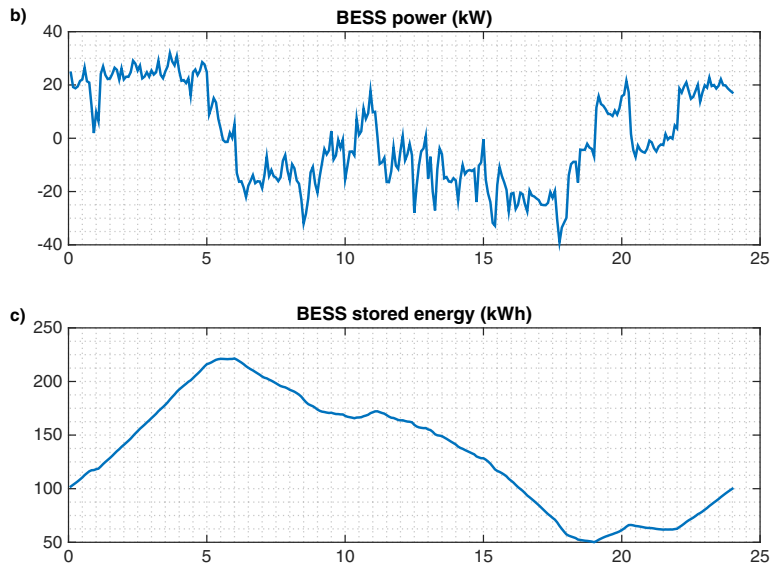

Figure 3. BESS operation in a $24 \mathrm{~h}$ scenario: a) profile of the feeder consumption without the BESS (red) and with the BESS (blue); b) Power injection of the BESS c) Energy stored in the BESS.

Table II

AGEING ASSESSMENT FOR A LOAD LEVELING STRATEGY WITH DIFFERENT SCENARIOS

\begin{tabular}{lll}
\hline$E_{\text {exch }}$ & $N_{c}$ & EOL \\
\hline $433.18 \mathrm{kWh}$ & 1.36 & 40.36 years \\
477.08 & 1.49 & 36.68 \\
505.91 & 1.58 & 34.58 \\
513.19 & 1.61 & 34.09 \\
313.34 & 0.98 & 55.82 \\
525.7 & 1.65 & 33.29 \\
337.03 & 1.06 & 51.92 \\
505.54 & 1.58 & 34.59 \\
437.79 & 1.37 & 39.95 \\
\hline
\end{tabular}

variances of the feeder consumption and of the total power absorption including the BESS contribution. These values measure the performance of the load leveling objective: it can be seen how $\operatorname{var}(P)$ is several times smaller of the variance of the consumption profile $\operatorname{var}(L)$. Table III reports as well the values obtained for the average daily exchanged energy $E_{\text {exch }}$, number of equivalent cycles $N_{c}$ and the expected EOL for the case analyzed. The expected cycling related battery lifetime for the proposed load leveling strategy is of about 40 years. This number being considerably higher of the calendar lifetime of the cells, i.e. 20 years, illustrates that for the specific application and cell chemistry the cycling ageing is not the main source of ageing, and therefore there is no need to limit the load leveling operation of the BESS with regard to this
Table III

PERFORMANCES FOR UNCONSTRAINED BESS OPERATION EMPLOYING CELLS WITH 20000 CYCLES

\begin{tabular}{ll}
\hline Metric & Value \\
\hline $\operatorname{var}(L)$ & $1.79 \cdot 10^{3} \mathrm{~kW}^{2}$ \\
$\operatorname{var}(P)$ & $396.15 \mathrm{~kW}^{2}$ \\
$E_{\text {exch }}$ & $789 \mathrm{kWh}$ \\
average $N_{c}$ & 1.41 \\
expected EOL due to cycling & 40.1 years \\
expected calendar EOL & 20 years \\
\hline
\end{tabular}

aspect, in order to be able to reach the expected calendar lifetime of 20 years.

\section{Implementation of an additional constraint in the BESS control strategy}

In order to take into account also for the degradation of the cells, the following additional constraint, which bounds the exchanged energy $E_{\text {exch }}$ of the BESS, can be added to the control problem described by Eq. 7-10:

$$
\frac{1}{2 E_{\text {batt }}} \frac{\Delta T}{3600} \sum_{j=0}^{i}\left|w_{j} B_{j}\right|<N_{M}
$$

Where $N_{M}$ is a number of cycles per day chosen in relation to a desired end of life time $E O L$ in years.

To quantify the usefulness of the additional constraint, we consider a cell chemistry having a cycling lifetime of 3500 cycles and a calendar one of 10 years, these values being typical for classical li-ion cell chemistries. We compare the simulation results obtained operating the battery disregarding the ageing limitation and considering it. Figure 4 reports the feeder consumption profiles, BESS power injection and BESS stored energy for a day of operation without the contribution of the BESS and in the two considered cases. By measuring the exchanged energy and average equivalent cycles per day, we see how with such cells, the expected EOL of the BESS is reached after 7 years. This case illustrates the importance to integrate the cycling ageing into the control strategy, as the EOL is reached before the calendar lifetime declared by the manufacturer. Table IV reports the relevant metrics for this case. We set then an additional constraint to the load leveling minimization algorithm, such that the battery EOL occurs after 10 years. We can see in Table $\mathrm{V}$ that by applying this constraint the performances with regard to load leveling are worsened, but the lifetime goal is reached. The ageing constraint is activated in the $55 \%$ of the days, whereas in the $45 \%$ of the case the BESS operation is not limited by the ageing constraint.

\section{CONCLUSIONS}

A novel method to assess ageing of electrochemical cells utilized in stationary BESS is proposed. This method is then utilized to analyze a load leveling strategy for a $280 \mathrm{kWh}$ lithium-titanate BESS connected on a medium voltage feeder and to develop and evaluate an ageing aware control. 
Table IV

PERFORMANCES FOR UNCONSTRAINED BESS OPERATION AND EMPLOYING CELLS WITH 5000 CYCLES

\begin{tabular}{ll}
\hline Metric & Value \\
\hline $\operatorname{var}(P)$ & $415.81 \mathrm{~kW}^{2}$ \\
$E_{\text {exch }}$ & $789 \mathrm{kWh}$ \\
$N_{c}$ & 1.41 \\
expected EOL due to cycling & 7 years \\
expected calendar EOL & 10 years \\
\hline
\end{tabular}

Table V

PERFORMANCES FOR BESS OPERATION CONSTRAINED TO A LIFETIME OF 30 YEARS AND EMPLOYING CELLS WITH 5000 CYCLES

\begin{tabular}{ll}
\hline Metric & Value \\
\hline $\operatorname{var}(P)$ & $685.83 \mathrm{~kW}^{2}$ \\
$E_{\text {exch }}$ & $401 \mathrm{kWh}$ \\
$N_{c}$ & 0.96 \\
expected EOL due to cycling & 10 years \\
expected calendar EOL & 10 years \\
\hline
\end{tabular}

a)

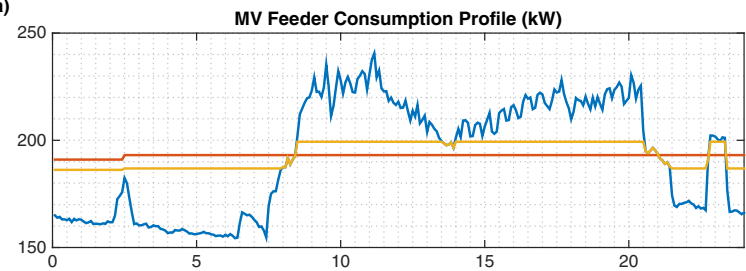

b)
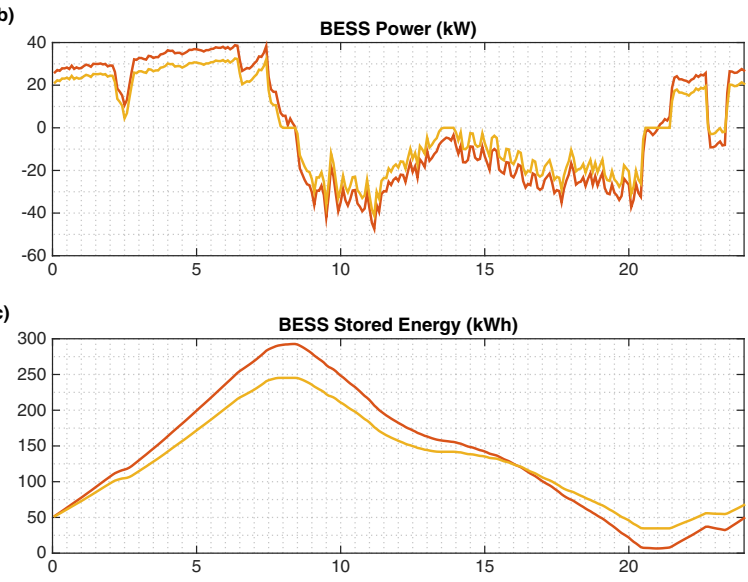

Figure 4. Comparison of a load leveling daily profile without BESS operation (blue), with BESS operating without ageing constraint (red) and with BESS operating with EOL constrained to 30 years (yellow).
It is shown how, a cycling lifetime of 20000 cycles allows for a BESS cycling lifetime of over 40 years, thus constituting a suitable value for a grid level installation. Moreover, it is quantified the effect of adding a constraint related to the minimization of ageing phenomena on the fulfillment of the load leveling control objective for a li-ion BESS. For a BESS composed by cells capable of 3500 cycles, the BESS lifetime can be extended from 7 to about 10 years but accepting a limitation on the load leveling performance. In the proposed case study, the variance of the daily consumption profile of the MV feeder passes from $415 \mathrm{~kW}^{2}$ to $686 \mathrm{~kW}^{2}$.

The proposed method can be used to determine, for a given BESS application, the minimum number of cycles that the cells need to be able to sustain in order to achieve a desired lifetime. It can be moreover used to assess the necessary tradeoff between BESS lifetime and desired performance.

\section{REFERENCES}

[1] K. Divya and J. Østergaard, "Battery energy storage technology for power systems - an overview", Electric Power Systems Research, vol. 79, no. 4, pp. 511-520, 2009.

[2] G. D. Rodriguez, "A utility perspective of the role of energy storage in the smart grid", in Power and Energy Society General Meeting, 2010 IEEE, IEEE, 2010, pp. 1-2.

[3] J. Leadbetter and L. Swan, "Battery storage system for residential electricity peak demand shaving", Energy and buildings, vol. 55, pp. 685-692, 2012.

[4] A. Oudalov, R. Cherkaoui, and A. Beguin, "Sizing and optimal operation of battery energy storage system for peak shaving application", in Power Tech, 2007 IEEE Lausanne, IEEE, 2007, pp. 621-625.

[5] B. Belvedere, M. Bianchi, A. Borghetti, C. A. Nucci, M. Paolone, and A. Peretto, "A microcontroller-based power management system for standalone microgrids with hybrid power supply", Sustainable Energy, IEEE Transactions on, vol. 3, no. 3, pp. 422-431, 2012.

[6] F. Sossan, D. Torregrossa, E. Namor, R. Cherkaoui, and M. Paolone, "Control of a battery energy storage system accounting for the charge redistribution effect to dispatch the operation of a medium voltage feeder", in PowerTech, 2015 IEEE Eindhoven, IEEE, 2015, pp. 1-6.

[7] T. Reddy and L. David, Linden's Handbook of Batteries, 4th edition. McGraw-Hill Education, 2010.

[8] J. Christensen, V. Srinivasan, and J. Newman, "Optimization of lithium titanate electrodes for high-power cells", Journal of The Electrochemical Society, vol. 153, no. 3, A560-A565, 2006.

[9] M. Koller, T. Borsche, A. Ulbig, and G. Andersson, "Defining a degradation cost function for optimal control of a battery energy storage system", in PowerTech (POWERTECH), 2013 IEEE Grenoble, IEEE, 2013, pp. 1-6. 
[10] P. Haessig, H. Ben Ahmed, and B. Multon, "Energy storage control with aging limitation", in PowerTech, 2015 IEEE Eindhoven, IEEE, 2015, pp. 1-6.

[11] J. Vetter, P. Novák, M. Wagner, C. Veit, K.-C. Möller, J. Besenhard, M. Winter, M. Wohlfahrt-Mehrens, C. Vogler, and A. Hammouche, "Ageing mechanisms in lithium-ion batteries", Journal of power sources, vol. 147, no. 1, pp. 269-281, 2005.

[12] D. U. Sauer and H. Wenzl, "Comparison of different approaches for lifetime prediction of electrochemical systems-using lead-acid batteries as example", Journal of Power Sources, vol. 176, no. 2, pp. 534-546, 2008.

[13] M. Safari, M. Morcrette, A. Teyssot, and C. Delacourt, "Multimodal physics-based aging model for life prediction of li-ion batteries", Journal of The Electrochemical Society, vol. 156, no. 3, A145-A153, 2009.

[14] A. Nourai, V. Kogan, and C. M. Schafer, "Load leveling reduces t\&d line losses", Power Delivery, IEEE Transactions on, vol. 23, no. 4, pp. 2168-2173, 2008.

[15] I. Papič, "Simulation model for discharging a leadacid battery energy storage system for load leveling", Energy Conversion, IEEE Transactions on, vol. 21, no. 2, pp. 608-615, 2006.

[16] C. H. Lo and M. D. Anderson, "Economic dispatch and optimal sizing of battery energy storage systems in utility load-leveling operations", IEEE Transactions on Energy Conversion, vol. 14, no. 3, pp. 824-829, 1999.

[17] K. Mamadou, E. Lemaire, A. Delaille, D. Riu, S. Hing, and Y. Bultel, "Definition of a state-of-energy indicator (soe) for electrochemical storage devices: Application for energetic availability forecasting", Journal of The Electrochemical Society, vol. 159, no. 8, A1298-A1307, 2012.

[18] M. Pignati, M. Popovic, S. Barreto, R. Cherkaoui, G. Dario Flores, J.-Y. Le Boudec, M. Mohiuddin, M. Paolone, P. Romano, S. Sarri, et al., "Real-time state estimation of the epfl-campus medium-voltage grid by using pmus", in Innovative Smart Grid Technologies Conference (ISGT), 2015 IEEE Power \& Energy Society, IEEE, 2015, pp. 1-5. 